\title{
The Effect on the Optical Absorption Coefficients due to the Positions in the Plane of Square GaAs / Al(GaAs) Quantum Well Wire under the Laser Field
}

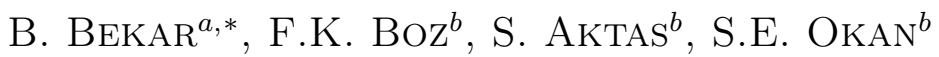 \\ ${ }^{a}$ Trakya University, Vocational of Kesan, 22800 Edirne, Turkey \\ ${ }^{b}$ Trakya University, Department of Physics, 22030 Edirne, Turkey
}

(Received March 15, 2019; revised version July 2, 2019; in final form September 30, 2019)

The laser field effects on the electronic and optical properties depending on two different positions in the plane of GaAs/Al (GaAs) quantum wire with square cross sections have been investigated. The electron energy levels and the electronic wave functions of the wires are calculated using the finite differences method within the effective mass approximation. The differences of the total absorption coefficients and the reflection index changes sourced from the cross-sectional shape were determined and explained with and without laser field. From the results the blue shift and red shift effects on the total absorption coefficients and the reflection index changes were identified depending on the laser field strength and the positions of square $\mathrm{GaAs} / \mathrm{Al}(\mathrm{GaAs})$ quantum well wire in the plane.

DOI: 10.12693/APhysPolA.136.882

PACS/topics: quantum well wire, intense laser field, absorption coefficient, refractive index changes

\section{Introduction}

Low-dimensional electronic devices such as photo detectors, electro-optical modulators, and far-infrared laser amplifiers are pioneering products of advanced semiconductor technologies. Studies on the electronic and optical properties of these quantum structures have consequently become an essential branch of the concerning research as a lot of work have been devoted to the determination of the transition energies, the absorption coefficients, and the refraction index changes [1-10]. It has been shown that the potential profile of structure strongly affects the linear and nonlinear optical absorption coefficients [1-3]. The role of external electric field on the electron location and the potential profile change created by a laser field was the subject of the coaxial wire research [3-7]. The wire containing parabolic and semi-parabolic quantum wells exhibits the properties that the variations of the resonance peaks increases with the increasing laser strength as they are shifted to lower energies [3-4]. In conjunction with the electric field, the width of the quantum well in the structure has been found to be a very effective parameter on the refractive index and absorption coefficients [4]. The photon energies of the total absorption coefficients exhibit the red shift as the hydrostatic pressure is increased while the blue shift is observed if the temperature is increased $[5,6]$. The radius of the dot, the incident optical intensity, the stoichiometric

*corresponding author; e-mail: bahadirbekar@trakya.edu.tr ratio of the constituent elements, and whether the dot is doped with the impurity or not have been proved to be characterizing parameters on the linear and the nonlinear $1 s-1 p, 1 p-1 d$, and $1 d-1 f$ transitions in a spherical quantum dot $[8,9]$. The photon energies of the optical absorption coefficients showed blue shift with the increasing diameters of the gold nano particle for the hybrid structure in which the metallic nano-particle was coupled with a multilayered quantum dot [10].

Quantum wires, which can be described as twodimensional quantum confinement of the electrons, are a class of low-dimensional structures and the determination of their optical properties are essentially achieved through the calculations of the allowed transition energies beforehand. The research on the optical properties of the quantum wires can be sampled as follows. The electronic states of different cross-sectional shaped quantum wires, namely the square, the circular, and the hexagonal cross-sectional wires, were calculated using Arnoldi algorithm and the finite differences method and it was shown that the energy levels are determined by the cross-sectional area of the wires $[11,12]$. Similarly, the infrared transition of an impurity atom in the cylindrical GaAs- $\mathrm{Ga}_{0.6} \mathrm{Al}_{0.4}$ As quantum well wires under an external magnetic field were shown to be depending upon geometrical confinement of the wire [13]. It was proved that the conductive subband structure of wire exhibits the blue shift effect by non-resonant transverse laser field [14].

The finite differences method was once again used to calculate the energy levels and the wave functions in a cylindrical quantum well wire under the high intensity laser field, which deforms the wire potential and 
causes anisotropic linear and nonlinear absorptions of light propagating in the $x-y$ plane [15]. The influence of the tilted angle which was defined as the angle between the laser field polarization direction and the cylindrical quantum well wire axis has been found to cause the complete deformation of the cylindrical symmetry of the quantum confinement potential and breaking down the degeneracy of the electronic states [16]. The optical absorption coefficients and refractive index changes in a two-dimensional system are strongly controlled with the incident optical intensity and the carrier density of a two-dimensional system [17].

Duque et al. have presented the results of the linear and nonlinear optical absorption and relative refractive index change associated with $1 s \rightarrow 2 p$ transitions between impurity states in triangular cross-sectioned quantum-well wires for both the $x$-polarization and $y$-polarization of the indicent light. In these structures, the calculations showed a mixed effect of red shift and blue shift depending on the location of the donor atom [18]. The magnetic field effect on the third harmonic generation in quantum well wires with triangular cross-section has been studied by Niculescu et al. They found that an axial orientation of the field allows the third harmonic generation and the third-order nonlinear susceptibility strongly depends on the polarization of the incident light [19]. The laser field effects on the intersubband optical absorption and the refractive index changes in GaAs/AlGaAs quantum wire with equilateral triangle cross section have also been investigated. The subband states were calculated by using a finite element method. It' has been found that the laser field allows an efficient red shift tuning of the $x$-polarized light absorption at high values of laser parameter and a blue shift tuning of the $y$-polarized light absorption for lower values of the laser intensity [20].

Pimenta et al. have published an article dealing with linear and nonlinear optical properties of single polytypic GaAs nanowires. They emphasized the importance to the crystallography of nanowires in optical properties [21]. In literature, studies on optic properties of cylindrical and triangle cross section quantum wires are seen, but electronic properties of ground state in the square cross section quantum wires are generally investigated under external fields [22-27]. It has been observed that the binding energy and the optical transition of the exciton are dependent on the sizes of the rectangular quantum well wire under intense laser field and magnetic field [28].

The first time scope, to the best of our knowledge, of the present study is to investigate the electronic and optical properties for two different positions of a square $\mathrm{GaAs} / \mathrm{Al}(\mathrm{GaAs})$ quantum well wire (SQW) with the laser polarization direction. The structure of work is as follows: Sect. 2 describes the theoretical model. The influence of the laser field on an electron in two different SQW are investigated, and the total absorption coefficients and the refractive index changes are presented in Sect. 3. Finally, Sect. 4 contains the summarized of the results of the study.

\section{Theory}

Let us consider a square cross-sectioned quantum well wire basically formed by GaAs rod on the $z$-axis enveloped by an Al (GaAs) layer. We named two different positions of the SQW with the laser polarization direction as if they are different positions of the SQWs. They are SQWx0 and SQWx45, where $x$ is the laser polarization direction as 0 and 45 the angle between the $x$-axis of the wire and the laser polarization. The time-dependent Schrödinger equation, in the effective-mass approximation, can be written as [20]:

$$
\begin{aligned}
& \left\{\frac{1}{2 m^{*}}\left[\boldsymbol{p}_{\perp}+e \boldsymbol{A}(t)\right]^{2}+V(x, y)\right\} \psi(x, y, t)= \\
& \mathrm{i} \hbar \frac{\partial}{\partial t} \psi(x, y, t)
\end{aligned}
$$

where $\boldsymbol{p}_{\perp}$ is the momentum of the electron on the $(x, y)$ plane, $\mathrm{m}^{*}$ is the effective mass of the electron, $e$ is the electron charge when the laser field is off, the confinement potential $V(x, y)$ in Eq. (1) is given by

$$
\begin{aligned}
& V(x, y)=V_{S}(x, y)= \\
& \begin{cases}0, & \text { if }(x, y) \in\left\{(x, y) \in R^{2}|| x\left|\leq \frac{L}{2},\right| y \mid \leq \frac{L}{2}\right\} \\
V_{0}, & \text { otherwise }\end{cases}
\end{aligned}
$$

for the SQWx0, and

$$
\begin{aligned}
& V(x, y)=V_{D}(x, y)= \\
& \begin{cases}0, & \text { if }(x, y) \in\left\{(x, y) \in R^{2}|| x|+| y \mid \leq \frac{L}{\sqrt{2}}\right\}, \\
V_{0}, & \text { otherwise }\end{cases}
\end{aligned}
$$

for the SQWx45 $V_{0}$ in Eqs. (2) and (3) is the conduction band offset between GaAs and $\mathrm{Al}$ (GaAs). The laser vector potential is given as $\boldsymbol{A}(t)=\hat{x} A_{0} \cos (w t)$, $\hat{x}$ being the unit vector along the $x$-axis. By applying the time-dependent translation $x \rightarrow x+\alpha_{0} \sin (w t)$ and performing the unitary Kramers-Henneberger transformation [20, 29-37], Eq. (1) can be rewritten as

$$
\left\{\frac{\boldsymbol{p}_{\perp}}{2 m^{*}}+\tilde{V}(x, y, t)\right\} \tilde{\psi}(x, y, t)=\mathrm{i} \hbar \frac{\partial}{\partial t} \tilde{\psi}(x, y, t)
$$

where $\tilde{V}(x, y, t)$ is the laser-dressed confinement potential. In the high-frequency limit [30], the timeindependent Schrödinger equation is

$\left[-\frac{\hbar^{2}}{2 m *}\left(\frac{\partial^{2}}{\partial x^{2}}+\frac{\partial^{2}}{\partial y^{2}}\right)+\tilde{V}_{D}(x, y)\right] \tilde{\psi}(x, y)=E \tilde{\psi}(x, y)$

where the averaged dressed potential energy $\tilde{V}_{D}(x, y)$ is given by

$$
\begin{gathered}
\tilde{V}_{D}(x, y)=\frac{\omega_{D}}{2 \pi} \int_{0}^{2 \pi / \omega_{D}} \tilde{V}(x, y, t) \mathrm{d} t= \\
\frac{1}{2 \pi} \int_{0}^{2 \pi} V\left(x+\alpha_{0} \sin \varphi, y\right) \mathrm{d} \varphi
\end{gathered}
$$


Here $2 \pi / \omega_{D}$ is the period of the laser field and $\alpha_{0}=e A_{0} / m^{*} \omega_{D}$ is the laser dressing parameter in Eq. (6) [30-35]. The first three energies and eigenfunctions of the electron with and without the laser field are numerically calculated by solving Eq. (5) with the bidimensional finite differences method.

The known energies and the known wave functions lead to the calculations of the refractive indexes and the absorption coefficients by using the density matrix method [5, 38-40]. In the electric dipole approximation, for $x$ - and $y$-polarization of the incident radiation, the elements of dipole transition matrix between initial state $(i)$ and final state $(f)$ is given by

$$
\begin{aligned}
& M_{i f}^{r=x, y}=\left\langle\tilde{\psi}_{i}|\operatorname{er}| \tilde{\psi}_{f}\right\rangle= \\
& e \int_{-\infty}^{+\infty} \int_{-\infty}^{+\infty} \tilde{\psi}_{i}^{*}(x, y) r \tilde{\psi}_{f}(x, y) \mathrm{d} x \mathrm{~d} y
\end{aligned}
$$

in addition $\tilde{\psi}_{i}$ and $\tilde{\psi}_{f}$ in Eq. (7) are the wave functions of initial and final states. Then, the linear and nonlinear optical absorption coefficients for the electronic polarization as a response to an electromagnetic field with frequency $\omega$ can be calculated by $[5,6,38-43]$ :

$$
\begin{aligned}
& \beta_{r}^{(1)}(\hbar \omega)=\frac{\sigma}{c \varepsilon_{0} n_{s}} \frac{\hbar \omega\left|M_{i f}^{r}\right|^{2} / \tau_{i n}}{\left(E_{i f}-\hbar \omega\right)^{2}+\left(\hbar / \tau_{i n}\right)^{2}}, \\
& \beta_{r}^{(3)}(\hbar \omega)=-\frac{2 \sigma}{\left(c \varepsilon_{0} n_{s}\right)^{2}} \frac{\hbar \omega I\left|M_{i f}^{r}\right|^{4} / \tau_{i n}}{\left[\left(E_{i f}-\hbar \omega\right)^{2}+\left(\hbar / \tau_{i n}\right)^{2}\right]^{2}} \\
& \times\left[1-\left|\frac{M_{f f}^{r}-M_{i i}^{r}}{2 M_{i f}^{r}}\right|^{2}\right. \\
& \left.\times \frac{\left(E_{i f}-\hbar \omega\right)^{2}-\left(\hbar / \tau_{i n}\right)^{2}+2 E_{i f}\left(E_{i f}-\hbar \omega\right)}{E_{i f}^{2}+\left(\hbar / \tau_{i n}\right)^{2}}\right],
\end{aligned}
$$

where $\varepsilon_{0}$ is the electric constant, $c$ is the speed of light in vacuum $n_{s}$ is the refractive index, $I$ is the optical intensity of the incident radiation, $\tau_{i n}$ is the intersubband relaxation time, and the transition energy $E_{i f}=E_{f}-E_{i}$ is the energy difference between the final and initial states. Neglecting the higher harmonic terms for quantum structures with particularly symmetric geometries, the total absorption coefficient $\beta_{r}(\hbar \omega, I)$ is given by

$$
\beta_{r}(\hbar \omega, I)=\beta_{r}^{(1)}(\hbar \omega)+\beta_{r}^{(3)}(\hbar \omega, I) .
$$

The linear and nonlinear contributions to refractive index changes are obtained as [18]:

$$
\begin{aligned}
& \frac{\Delta n_{r}^{(1)}(\hbar \omega)}{n_{s}}=\frac{\sigma}{2 \varepsilon_{0} n_{s}^{2}} \frac{\left|M_{i f}^{r}\right|^{2}\left(E_{i f}-\hbar \omega\right)}{\left(E_{i f}-\hbar \omega\right)^{2}+\left(\hbar / \tau_{i n}\right)^{2}} \\
& \frac{\Delta n_{r}^{(3)}(\hbar \omega, I)}{n_{s}}=-\frac{\sigma}{c \varepsilon_{0}^{2} n_{s}^{3}} \frac{I\left|M_{i f}^{r}\right|^{4}\left(E_{i f}-\hbar \omega\right)}{\left[\left(E_{i f}-\hbar \omega\right)^{2}+\left(\hbar / \tau_{i n}\right)^{2}\right]^{2}} \times
\end{aligned}
$$

$$
\begin{aligned}
& \times\left\{1-\left|\frac{M_{f f}^{r}-M_{i i}^{r}}{2 M_{i f}^{r}}\right|^{2}\right. \\
& \left.\times \frac{E_{i f}\left(E_{i f}-\hbar \omega\right)^{2}-\left(\hbar / \tau_{i n}\right)^{2}\left(3 E_{i f}-2 \hbar \omega\right)}{\left[E_{i f}^{2}+\left(\hbar / \tau_{i n}\right)^{2}\right]\left(E_{i f}-\hbar \omega\right)}\right\} .
\end{aligned}
$$

The total refractive index change is then given by

$$
\frac{\Delta n_{r}(\hbar \omega, I)}{n_{s}}=\frac{\Delta n_{r}^{(1)}(\hbar \omega)}{n_{s}}+\frac{\Delta n_{r}^{(3)}(\hbar \omega, I)}{n_{s}}
$$

\section{Results}

The electronic and the optical properties of the considered wires under a laser field are presented for the SQWx0 and SQWx45, which have the equal cross-sectional edge length of $L=105 \AA$. The barrier height constituted by the $\mathrm{Al}$ (GaAs) coating is taken to be $V_{0}=228 \mathrm{meV}$ and the effective mass is assumed constant in the whole structure, taken to be $m^{*}=0.0665 m_{0}[15,20]$. The other physical parameters used in the calculations can be lined up as the electron density $\sigma=3 \times 10^{22} \mathrm{~m}^{-3}$, the refractive index of GaAs, $n_{s}=3.2$ and the intersubband relaxation time, $\tau_{i n}=0.2 \mathrm{ps}[15,20]$. In the presence of the laser field, we use $\varepsilon=10.9 \varepsilon_{0}$, which corresponds to the high frequency dielectric constant for GaAs [15, 44, 45], instead of $\varepsilon=13.18 \varepsilon_{0}$ for the off-field case.

The potential profiles of SQWx0 and SQWx45 are shown for the laser strengths of $0,40 \AA$, and $80 \AA$ in Fig. 1. The confining potential in the upper part of the SQWx45 widens while the lower part narrows with the increasing strength of $x$-axis polarized laser field. The potential profile takes a rectangular shape at the top lying along the laser polarization direction for the SQWx0 while the shape at the top becomes a hexagon with the application of the laser field for SQWx45. Further increases in the strength widen the area at the surface of the potential profiles.

To imply the importance of the laser field on the energy levels, we present the first three levels of the subband energies for the SQWx0 and SQWx45 as functions of the laser field strength in Fig. 2a and b, respectively. The $E_{1}, E_{2}$, and $E_{3}$ energies of the both wires are equal in the absence of the laser field because these two wires can transform into each other by $45^{\circ}$ axial turn, and they have same cross-sectional area. However, the application of the laser field completely alters this occurrence by creating different energy behaviors in the wires. The increase in the $E_{1}$ due to the high fields in the SQWx45 is less than that in the SQWx0. The $E_{2}$ and $E_{3}$ energies are degenerate when the field is off. For the SQWx0, the non-degenerate splitting of these energy levels becomes distinguishable at about $\alpha_{0} \cong 5 \AA$, and they undergo anti-crossing at $\alpha_{0} \cong 49 \AA$, where the non-degenerate separation between these values reaches a minimum value, and then starts to increase again, as can be seen in Fig. 2a [46, 47]. For the stronger 
(a)
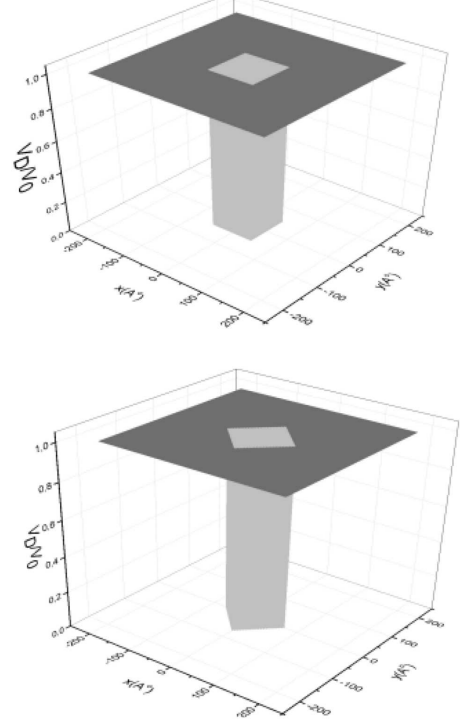

(b)
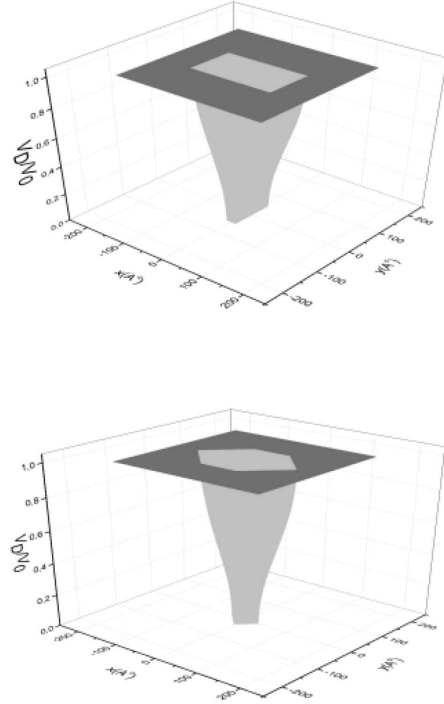

(c)
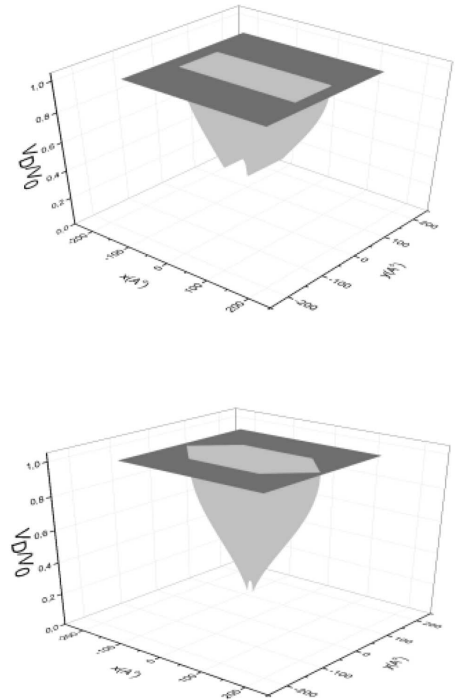

Fig. 1. Laser-dressed potential profiles of the GaAs/Al (GaAs) SQWx0 (top) and SQWx45 (bottom) for the laser strengths of (a) $\alpha_{0}=0$, (b) $\alpha_{0}=40 \AA$, (c) $\alpha_{0}=80 \AA$.
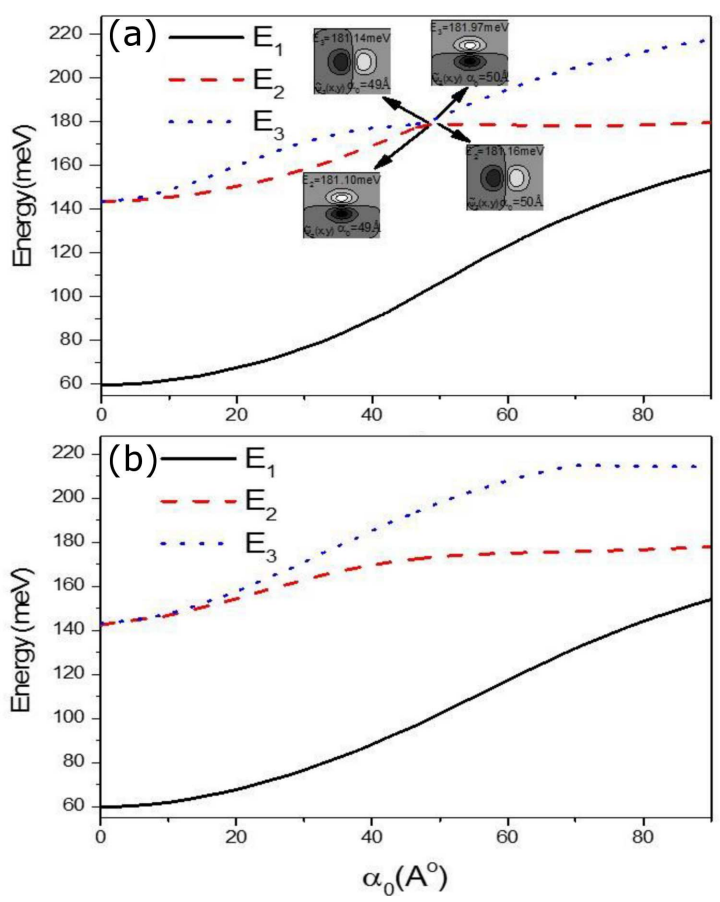

Fig. 2. Energies of lowest three electronic states as a function the laser dressing parameter for the $\mathrm{GaAs} / \mathrm{Al}$ (GaAs) square quantum well wires for (a) SQWx0, and (b) SQWx45. The icons in part (a) are two-dimensional wave functions of the electron.

strengths than $\alpha_{0} \cong 49 \AA$, the $E_{2}$ reaches a plateau against the increasing $E_{3}$. Around the specific value of $\alpha_{0}$, these changes may be better understood in terms of the two-dimensional wave functions by comparing the gray scale icon in Fig. 2a. At the specific value of $\alpha_{0}=49 \AA$ for the SQWx0, the extremums of the $\tilde{\psi}_{2}(x, y)$ align on $y$-axis while the extremums of $\tilde{\psi}_{3}(x, y)$ align on the $x$-axis. It is vice versa with $\alpha_{0}=50 \AA$. All wave functions of $E_{2}$ to $E_{3}$ states for given values of a laser field strength reflect the orthogonality condition.

The behavior of $E_{2}$ and $E_{3}$ in the SQWx0 is different than in the SQWx45, as seen in Fig. 2b. For the SQWx45, the non-degenerate splitting of these energy levels becomes distinguishable at about $\alpha_{0} \cong 20 \AA$. The difference between the $E_{2}$ and $E_{3}$ increases up to $\alpha_{0} \cong 60 \AA$ and then remains constant. That is because the $E_{2}$ reaches a plateau for the fields stronger than $\alpha_{0} \cong 40 \AA$, as does $E_{3}$ for the fields stronger than $\alpha_{0} \cong 60 \AA$ in the SQWx 45 . The reason for these behaviors of $E_{2}$ and $E_{3}$ energies in the SQWx45 is the location in the $x-y$ plane of the SQWx0 under the laser field. This shows us that the position on the $x-y$ plane of the square quantum well wire is important under the laser field.

The optical properties of the SQWx0 and SQWx45 were determined by calculating the total absorption coefficients and the refraction index changes for $x$-polarized and $y$-polarized lights with an incoming intensity of $I=0.3 \mathrm{MW} / \mathrm{cm}^{2}$, which provides more pronounced results in comparison with the smaller intensity.

Figure $3 \mathrm{a}$ and $\mathrm{b}$ show the total absorption coefficients for the transition 1-2 of $y$-polarized and the transition 1-3 of $x$-polarized incident lights in the SQWx0 under $\alpha_{0}=0,20,40$, and $60 \AA$ laser strength values, respectively. As the total absorption coefficient depends on the elements of dipole transition matrix and the transition energies, the absorption appears at $88 \mathrm{meV}$ photon energy for both $x$ - and $y$ - polarization when the field is off. This is expected, because $E_{2}$ and $E_{3}$ are degenerate 

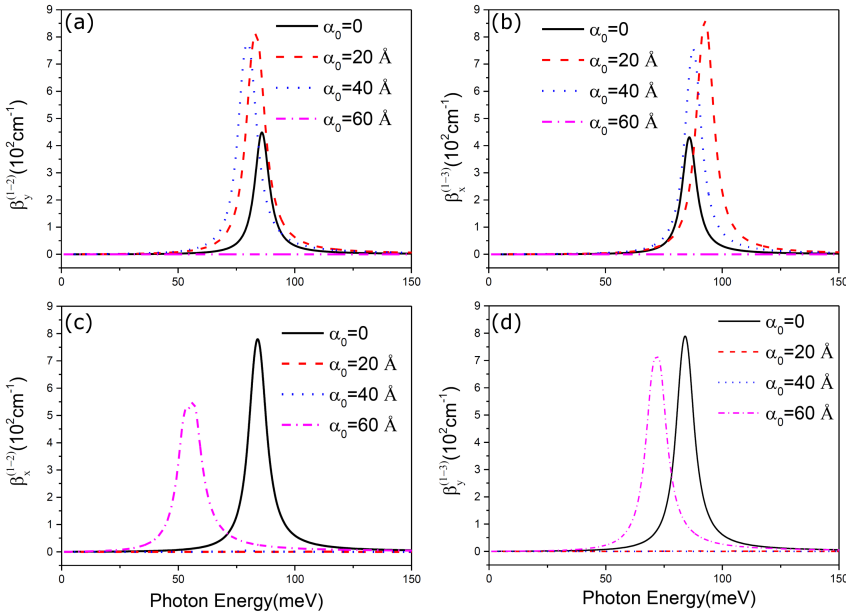

Fig. 3. Total absorption coefficients depending on photon energies under different strengths of the laser field: (a, c) 1-2 transitions with $y$-polarized and $x$-polarized lights in SQWx0, (b, d) 1-3 transitions with $x$-polarized and $y$-polarized lights in SQWx0.

if $\alpha_{0}=0$, and $\tilde{\psi}_{2}$ and $\tilde{\psi}_{3}$ are anti-symmetric wave functions. The photon energy shifts to lower energies with the increasing strength of the field for the $y$-polarized light as it is vice versa for the $x$-polarized light. The $1-3$ transitions with the $x$-polarized light shows that the photon energy shifts back to closer value of no-field case for $\alpha_{0}=40 \AA$ in comparison with the photon energy of $\alpha_{0}=20 \AA$ application. For $\alpha_{0} \geq 50 \AA$, there is no 1-2 absorption of the $y$-polarized light and 1-3 absorption of the $x$-polarized light in SQWx0 due to zero matrix elements.

Figures $3 \mathrm{c}$ and $3 \mathrm{~d}$ show the total absorption coefficients for the transition 1-2 of $x$-polarized and the transition $1-3$ of $y$-polarized incident lights in the SQWx0 under $\alpha_{0}=0,20,40$, and $60 \AA$ laser strength values, respectively. The total absorption coefficient appears at the same photon energies for both $x$ - and $y$ - polarization when the field is off. For $\alpha_{0}<50 \AA$, the 1-2 absorption of $x$-polarized light and the 1-3 absorption of $y$-polarized light are forbidden due to the zero elements of dipole transition matrix. On the other hand, the transitions of both polarized direction is allowed for $\alpha_{0} \geq 50 \AA$, the absorption peaks show redshifts increasing laser strength value. The reason for this is that elements of dipole transition matrix is different from zero by changing the wave functions under the influence of the laser field.

We show the results of the total absorption coefficient for SQWx45 in Fig. $4 a$ and b, and for SQWx0 as presented in Fig. 3c and d. For the $x$-polarized light, $\alpha_{0}=20 \AA$ causes the photon energy shifting to higher values in comparison with that of no-field case while it is at the lower values for $\alpha_{0}=40 \AA$ than the no-field absorption energy. The high field value $\alpha_{0}=60 \AA$ has nonzero transition matrix elements and gives an absorption in quite low energy in comparison with that of the other

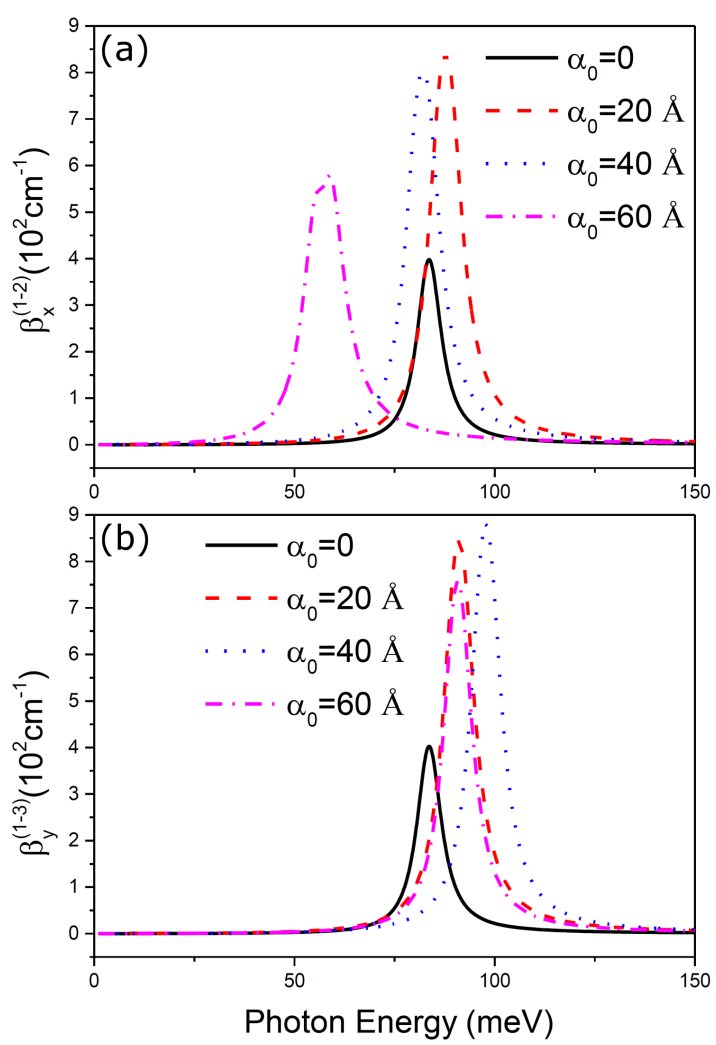

Fig. 4. Total absorption coefficients depending on photon energies under different strengths of the laser field for (a) $x$-polarized and (b) $y$-polarized lights in SQWx45.

strengths. A blue shift is observed in the absorption coefficients up to $\alpha_{0}=40 \AA$ with the $y$-polarized incident light, and then it shifts back to close values of no-field case for $\alpha_{0}=60 \AA$.

The refraction index changes for $x$ - and $y$-polarized lights are shown as a function of the incident photon energy with and without laser field in Fig. 5 for the SQWx0. The oscillation amplitudes of the refraction index changes increased with the laser field application. The general behavior pattern of the refraction index changes are almost same as the behavior pattern of the corresponding total absorption coefficients discussed earlier. In the Fig. 5a, the red shift of the refraction index changes occurs for $y$-polarized light up to $\alpha_{0}=40 \AA$ against the fact that the transition is forbidden for $\alpha_{0}=60 \AA$. On the other, the red shift of the 1-2 and 1-3 refraction index changes occurs for $x$ - and $y$-polarized lights, as seen in Fig. 5c and d.

The changes of refraction index depending on photon energies for different strengths of laser fields are shown in Fig. 6a and b for $x$-polarized and $y$-polarized lights in SQWx45. The general behavior of the refraction index changes is almost same with the behavior of the previous examples discussed earlier. In the SQWx45, the amplitude of the refraction index change for $\alpha_{0}=60 \AA$ in Fig. 6a is bigger than the amplitude for the other fields 

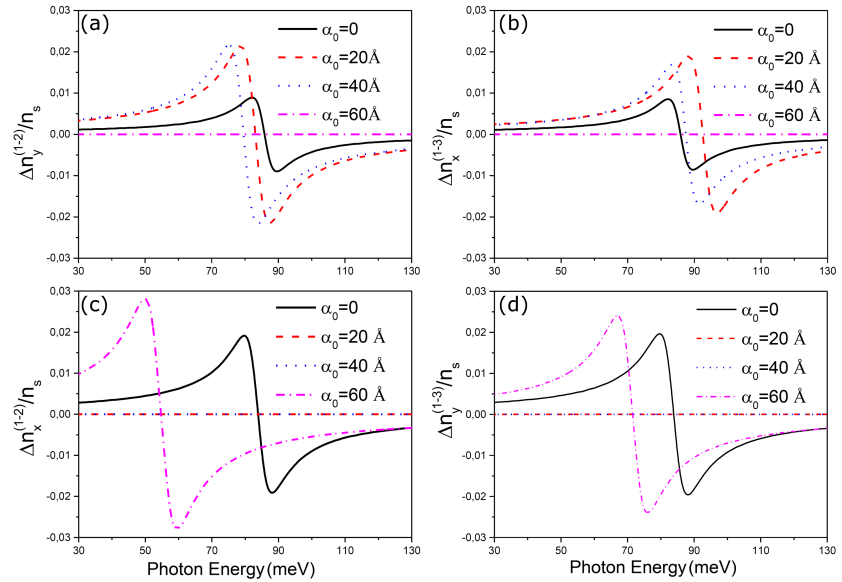

Fig. 5. Refraction index changes depending on photon energies for different strengths of laser fields: $(\mathrm{a}, \mathrm{c}$ ) 1-2 transitions with $y$-polarized and $x$-polarized lights in SQWx0, (b, d) 1-3 transitions with $x$-polarized and $y$-polarized lights in SQWx0.
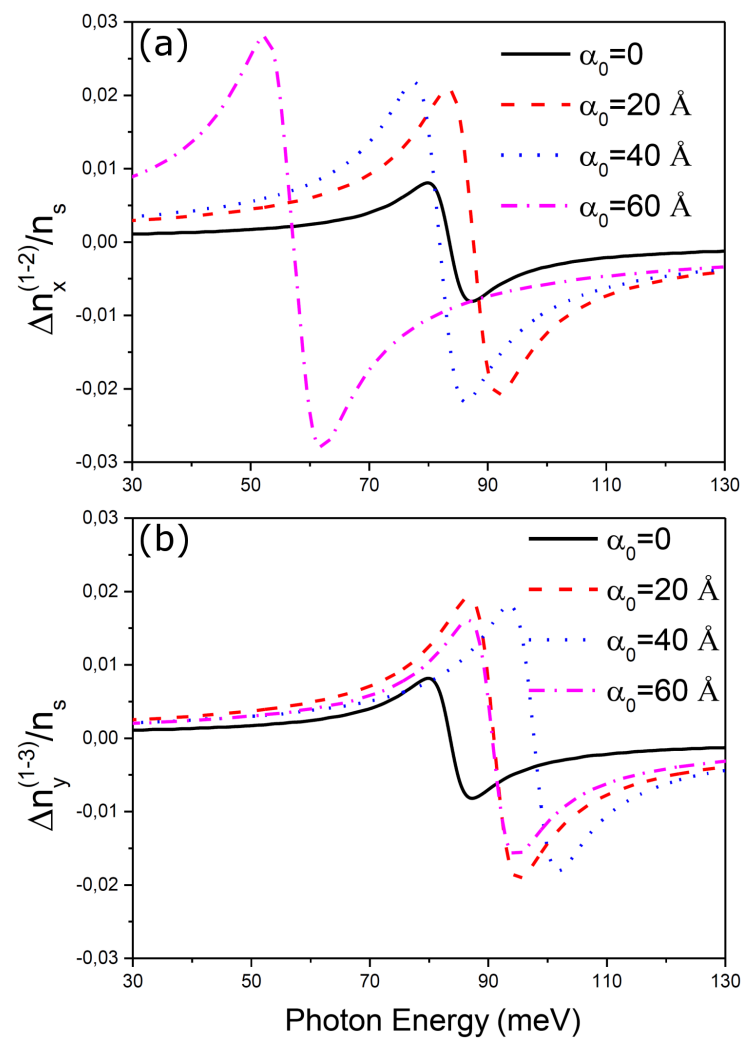

Fig. 6. Refraction index changes depending on photon energies for different strengths of laser fields: (a) $x$-polarized and (b) $y$-polarized lights in SQWx45.

and no field. This opposes to the observation on the total absorption coefficient with $x$-polarized light described in Fig. 4a. For the $y$-polarized light, a blue shift is observed in the refraction index changes up to $\alpha_{0}=40 \AA$. The bigger field for namely $\alpha_{0}=60 \AA$, causes a shift in the refraction index change to lower energies.

\section{Conclusions}

The electronic and optical properties for two different positions in the $x-y$ plane of square $\mathrm{GaAs} / \mathrm{Al}(\mathrm{GaAs})$ quantum wire under the laser field have been investigated theoretically. The wire considered was a GaAs/ $\mathrm{Al}$ (GaAs), for which the electronic wave functions and the energy levels were calculated under a laser field. The finite differences method was employed in the calculations. The position on the $x-y$ plane of the wire strongly affects the energy levels depending on the laser field parameter. The total absorption coefficients and the refraction index changes of the lowest three energy levels were calculated for the different laser field strengths.

The results showed that the SQWx0 allows the 1-3 absorption of the $x$-polarized light and the 1-2 absorption of the $y$-polarized light up to the critical laser field value $\alpha_{0} \cong 49 \AA$ as the $1-2$ absorption of the $x$-polarized light and the 1-3 absorption of the $y$-polarized light are allowed for $\alpha \geq 50 \AA$ fields With SQWx45, the 1-2 absorption of the $x$-polarized light and the 1-3 absorption of the $y$-polarized light are observed. The total absorption coefficients and the refraction index changes depending on photon energies for different strengths of laser fields was found to be important for the position of square quantum wire in the $x-y$ plane. These results can be used as a guide for circuit elements based on intersubband transitions in square quantum wire.

\section{References}

[1] B. Chen, K.-X. Guo, R.-Z. Wang, Z.-H. Zhang, Z.-L. Liu, Solid State Commun. 149, 310 (2009).

[2] A. Keshavarz, M.J. Karimi, Phys. Lett. A 374, 2675 (2010).

[3] E. Kasapoglu, C.A. Duque, H. Sari, I. Sökmen, Eur. Phys. J. B 82, 13 (2011).

[4] U. Yesilgul, F. Ungan, S. Sakiroglu, M.E. MoraRamos, C.A. Duque, E. Kasapoglu, H. Sarı, I. Sökmen, J. Lumin. 145, 379 (2014).

[5] F. Ungan, U. Yesilgul, E. Kasapoglu, H. Sari, I. Sökmen, Opt. Commun. 285, 373 (2012).

[6] E. Ozturk, I. Sokmen, J. Lumin. 134, 42 (2013).

[7] S. Panda, B.K. Panda, Superlattices Microstruct. 73 160 (2014).

[8] B. Çakır, Y. Yakar, A. Özmen, Prog. Electromagn. Res. M 21, 77 (2011).

[9] B.Çakır, Y. Yakar, A. Ozmen, J. Lumin. 132, 2659 (2012).

[10] N. Zamani, A. Keshavarz, H. Nadgaran, Physica B 490, 57 (2016).

[11] R. Khordad, H. Bahramiyan, Superlattices Microstruct. 88, 651 (2015).

[12] Z. Avazzadeh, R. Khordad, H. Bahramiyan, S.A. Mohammadi, J. Comput. Electron. $\mathbf{1 5}$ 931 (2016).

[13] P. Villamil, C. Cabra, N. Porras-Montenegro, Microelectron. J. 36, 383 (2005). 
[14] A. Radu, Physica E 44, 1446 (2012).

[15] E.C. Niculescu, L.M. Burileanu, A. Radu, A. Lupaşcu, J. Lumin. 131, 1113 (2011).

[16] A. Radu, E. C. Niculescu, J. Lumin. 132, 1420 (2012).

[17] C. Zhang, Z. Wang, M. Gu, Y. Liu, K. Guo, Physica $B$ 405, 4366 (2010).

[18] C.A. Duque, M.E. Mora-Ramos, E. Kasapoglu, F. Ungan, U. Yesilgul, S. Sakiroglu, H. Sari, I. Sökmen J. Lumin. 143, 304 (2013).

[19] E.C. Niculescu, M. Cristea, A. Radu, Physica E 57, 138 (2014).

[20] M.G. Barseghyan, C.A. Duque, E.C. Niculescu, A. Radu, Superlattices Microstruct. 66, 10 (2014).

[21] A.C.S. Pimenta, D.C. Teles Ferreira, D.B. Roa, M.V.B. Moreira, A.G. de Oliveira, J.C. González, M. De Giorgi, D. Sanvitto, F.M. Matinaga, J. Phys. Chem. C 120, 17046 (2016)

[22] Ulhas S. Sonawane, E.P. Samuel, C.K. Kasar, D.S. Patil, Optik 137, 115 (2017).

[23] M. Koksal, E. Kilicarslan, H. Sari, I. Sokmen, Physica B 404, 3850 (2009).

[24] G. Rezaei, S. Mousavi, E. Sadeghi, Physica B 407, 2637 (2012)

[25] A. Bilekkaya, S. Aktas, S.E. Okan, F.K. Boz, Superlattices Microstruct. 44, 96 (2008).

[26] S. Aktas, F.K. Boz, A. Bilekkaya, S.E. Okan, Physica E 41, 1572 (2009).

[27] C.A. Duque, M.E. Mora-Ramos, U. Yesılgul, F. Ungan, S. Sakıroglu, E. Kasapoglu, H. Sarı, I. Sökmen, Acta Phys. Pol. A, 125, 198 (2014).

[28] E. Kasapoglu, H. Sarı, M. Güneş, I. Sökmen, Surf. Rev. Lett. 11, 403 (2004).

[29] H. Kramers, in: Collected Scientific Papers, NorthHolland, Amsterdam, 1956, p. 866.

[30] M. Gavrila, J.Z. Kaminski, Phys. Rev. Lett. 52, 613 (1984).
[31] M. Gavrila, J. Phys. B: Phys. Rev. A: At. Mol. Opt. Phys. 35, R147 (2002).

[32] E.C. Valadares, Phys. Rev. B: Condens. Matter 41 $1282(1990)$

[33] E.C. Niculescu, L.M. Burileanu, A. Radu, Superlattices Microstruct. 44, 173 (2008).

[34] M. Marnescu, M. Gavrila, Phys. Rev. A: At. Mol. Opt. Phys. 53, 2513 (1995).

[35] C. Zhang, Appl. Phys. Lett. 78, 4187 (2001).

[36] M. Fujita, T. Toyoda, J.C. Cao, C. Zhang, Phys. Rev. B: Condens. Matter 67, 075105 (2003).

[37] F.K. Boz, S. Aktas, B. Bekar, S.E. Okan, Phys. Lett. A 376, 590 (2012)

[38] D. Ahn, S.L. Chuang, IEEE J. Quantum Electron. 23, 2196 (1987).

[39] S. Ünlü, İ. Karabulut, H. Şafak, Physica E 33(2006) 319

[40] E.M. Galdys, J.J. Shi, Phys. Status Solidi B 210, 237 (1998).

[41] M.R.K. Vahdani, G. Razei, Phys. Lett. A 373, 3079 (2009).

[42] T. Takagahara, Phys. Rev. B: Condens. Matter 36 , 99 (1987)

[43] E. Rosencher, Ph. Bois, Phys. Rev. B: Condens. Matter 44, 11315 (1991).

[44] F.M.S. Lima, M.A. Amato, L.S.F. Olavo, O.A.C. Nunes, A.L.A. Fonseca, E.F. da Silva, Phys. Rev. B: Condens. Matter 75, 073201 (2007).

[45] N. Eseanu, Phys. Lett. A 374, 1278 (2010).

[46] P. Harrison, Quantum Wells, Wires and Dots, 2nd ed. Wiley, New York, 2006.

[47] M.J. Karimi, G. Rezaei, J. Appl. Phys. 111, 064313 (2012). 